\title{
Epidemiology and Prevention of Fetal Death and Stillbirth
}

\author{
1Joachim W Dudenhausen, ${ }^{2}$ Amos Grünebaum
}

\begin{abstract}
In high-income countries, the stillbirth rate did not change during the last decades. For prevention of stillbirth we need an international consens about risk factors, causes and classification of stillbirth, national fetal registries and standadized protocols for stillbirth evaluation. The knowledge from these data may lead to further prevention strategies.
\end{abstract}

Keywords: Fetal autopsy, Fetal death, Placental histology, Stillbirth.

How to cite this article: Dudenhausen JW, Grünebaum A. Epidemiology and Prevention of Fetal Death and Stillbirth. Donald School J Ultrasound Obstet Gynecol 2016;10(2):160-162.

Source of support: Nil

Conflict of interest: None

\section{DEFINITION OF STILLBIRTH}

Internationally, there are many different definitions to define a stillbirth, most of these differences relate to the gestational age of the pregnancy or the fetal weight. According to the World Health Organization, ${ }^{1}$ a stillbirth is "... a baby born with no signs of life at or after 28 weeks' gestation" while according to the Centers for Disease Control a stillbirth is "... the death of a baby before or during delivery." 2

Some statistics of stillbirths exclude newborns with congenital anomalies or termination of pregnancy. These differences may have an influence at the precise rate of stillbirth, but the global trend will persist.

\section{INCIDENCE OF STILLBIRTH}

Worldwide, on average, six stillbirths occur each minute or nearly 3 million each year. ${ }^{3}$ The stillbirth rate has declined by $14 \%$ from 22.1 per 1,000 births in 1995 to 18.9 stillbirths per 1,000 births in 2009 , though there is a dearth of reliable data in regions where most stillbirths

\section{${ }^{1}$ Professor and Chairman Emeritus, ${ }^{2}$ Director}

${ }^{1}$ Department of Obstetrics, Charité - University Medicine Berlin Berlin, Germany

${ }^{2}$ Department of Obstetrics and Gynecology, Weill Cornell Medicine, New York, USA

Corresponding Author: Joachim W Dudenhausen, Professor and Chairman Emeritus, Department of Obstetrics, Charité University Medicine Berlin Augustenburger Platz 1, D 13353 Berlin, Germany, Phone: +493045057816 e-mail: Joachim. dudenhausen@charite.de occur. ${ }^{4}$ In high-income countries the stillbirth rate has remained constant for the past three decades, ranging from 3 to 5.3 per 1,000 births. $^{5}$

\section{RISK FACTORS FOR STILLBIRTH}

A systematic review and analysis for identifying major risk factors was suggested by Flenady et al. ${ }^{6}$ The more significant risk factors included maternal overweight and obesity, maternal smoking, small size for gestational age, placental abruption, preexisting maternal diabetes and hypertension, and primiparity.

Villamor and Cnattingius ${ }^{7}$ from Sweden reported the increasing risk with weight gain between pregnancies. Overweight and obesity increase the risk of diabetes type 2 and gestational diabetes, which increased the risk for stillbirths, too. The number of women with delayed motherhood is rising, specifically in high-income countries, which is leading to an increase of primiparous women older than 35 years. Stephansson et $\mathrm{al}^{8}$ found an odds ratio of 3.6 for stillborn risk in older primiparous women (>31 years). Waldenström et $\mathrm{al}^{9}$ described that maternal age is an independent risk factor for stillbirth in nulliparopus women. Pregnant women without or late antenatal care showed high odds ratios for stillbirth. Globally, low education level (8 years or fewer) and other low socioeconomic status measured by employment, marital status, and income increase the odds ratio for stillbirth clearly.

\section{CAUSE OF STILLBIRTH}

The topic of stillbirths especially in low-income countries (according to the World Bank countries classification) has received very little research or attention. Despite the large number of stillbirths worldwide, there is no standard international classification system for the cause of a stillbirth. Almost half of the deliveries in many developing countries occur at home, and underreporting of stillbirths is a significant problem. Reliable data about rates and causes are unavailable in many areas of the world. ${ }^{10}$ Higher and lower income countries have different stillbirth rates and causes, which often require different preventive strategies. ${ }^{11}$

The classification of fetal deaths is further complicated by the multiplicity of factors and pathophysiological processes regarding mother, fetus, and placenta. There are many classification systems published in the literature. ${ }^{12}$ 
Better than the pathoanatomical and clinical mixed classification system may be the two-step classification: (1) The indirect causes of fetal death as the result of autopsy and placental histology; (2) The risk factors from clinical reasons. ${ }^{13}$

In order to appropriately address whether the fetal death was caused spontaneously or as part of an induced termination of pregnancy - e.g., 55\% of all fetal deaths in 2010 in Canada were part of an induced termination of pregnancy $^{14}$ - fetal death registries are essential, partially to establish effective prevention strategies.

Prolonged and obstructed labor, preeclampsia, and various infections, all without adequate treatment, appear to account for the majority of stillbirths in developing countries. ${ }^{10}$

According to the Stillbirth Collaborative Research Network Writing Group, the majority of stillbirths had a probable or possible cause after a systemic evaluation, with obstetric conditions and placental anomalies among the most common causes, ${ }^{15}$ while in a Scandinavian study, two-thirds of all stillbirths were caused or were associated with placental pathology. ${ }^{16}$ Michalski et $\mathrm{al}^{17}$ studied nearly 1,500 stillbirths. Autopsy findings identified the cause of death in $46 \%$ of cases and gave new information in 51\%, which changed the estimated recurrence risk in $40 \%$ of cases. Hübner et $\mathrm{al}^{18}$ published a retrospective 8.5-year study of eight hospitals with clinical reports, data collection, interdisciplinary communication, and postmortem examination in the area of Bonn/Germany in which $52 \%$ were of a certain or uncertain cause of death.

\section{EVALUATION OF STILLBIRTH}

Optimally, all stillbirths should have a thorough evaluation done to confirm not only the cause of the stillbirth but also to recognize contributing factors. Several organizations have developed standardized protocols for stillbirth evaluation. ${ }^{11}$ Therefore, timely autopsy and placental histopathology performed by an experienced perinatal pathologist are crucial steps. Unfortunately, in low-income countries, access to perinatal pathologists is limited, and even in high-income countries they are not always available. The combined approach (clinical records including maternal laboratory evaluation, ${ }^{19}$ fetal autopsy, placental histology) will lead to a clarification of the cause in the majority of stillbirths. ${ }^{15,20}$ Inadequate postmortem examination will determine unexplained fetal deaths.

In cases when the parents do not accept the autopsy despite of compassionate counseling, minimally invasive approaches like radiological imaging, skeletal survey, or postmortem magnetic resonance imaging and blood sample collecting for cytogenetic are-partially-alternative methods of conventional autopsy. Nevertheless, appropriate counseling of the parents improve the autopsy rate.

\section{PLACENTAL FINDINGS}

Pinar et $\mathrm{al}^{21}$ compared placental lesions for 518 stillbirth cases and 1,200 live birth controls in a population-based study. They found significantly higher rates in stillbirth cases with single umbilical artery, velamentous cord insertion, diffuse terminal villous immaturity, inflammation like chorioamnionitis, vascular degenerative changes in chorionic plate, retroplacental hematoma, intraparenchymal thrombi, parenchymal infarction, fibrin deposition, fetal vascular thrombi, avascular villi, and placental hydrops.

\section{PREVENTION STRATEGY}

Searches in international peer-reviewed papers about stillbirth and some retrospective data collection in different areas of high-income countries showed the necessity of a standardized and complete postmortem procedure for development of effective prevention strategy. ${ }^{12}$

The raising of awareness and implementation of effective interventions for modifiable risk factors, such as overweight, obesity, maternal age, and smoking, are priorities for stillbirth prevention in high-income countries. ${ }^{7}$ Considering the evidence of obesity as one of the leading risk factors for stillbirth, strategies are needed to educate women in childbearing age about optimizing normal body mass index. Moreover, strategies against smoking and alcohol drinking, and promotion of smoking and alcohol cessation in pregnancy must be more effective.

Stillbirths can be further lowered by preconceptional treatments of medical disorders like diabetes, thyroid disorders, and hypertension. Therefore, preconceptional medical counseling is important for all women in childbearing age.

An advanced maternal age over 35 years especially in primiparous women contributes to the stillbirth rate of high-income countries. Strategies to decrease the mean maternal age are difficult to realize in our communities, but there are some policies for women with the decision for postponed motherhood. ${ }^{22}$

\section{CONCLUSION}

In low-income countries, better access to appropriate obstetric care, particularly during labor and delivery, and better screening and treatment of syphilis can reduce stillbirth rates dramatically, while in high-income countries, effective interventions for modifiable risk factors, such as overweight, obesity, maternal age, and smoking, are priorities for stillbirth prevention. 


\section{REFERENCES}

1. Available from: http://www.who.int/maternal_child_ adolescent/epidemiology/stillbirth/en/ [cited 2015 Dec 5].

2. Available from: http://www.cdc.gov/ncbddd/stillbirth/ facts.html [cited 2015 Dec 5].

3. Goldenberg RL, McClure EM, Bhutta ZA, Belizan JM, Rubens CE, Mabeya H, Flenady V, Darmstadt GL; Lancet's Stillbirths Series steering committee. Stillbirths: the vision for 2020. Lancet 2011 May 21;377(9779):1798-1805.

4. Cousens S, Blencowe H, Stanton C, Chou D, Ahmed S, Steinhardt L, Creanga AA, Tunçalp O, Balsara ZP, Gupta S, et al. National, regional, and worldwide estimates of stillbirth rates in 2009 with trends since 1995: a systematic analysis. Lancet 2011 Apr 26;377(9774): 1319-1330.

5. Reddy UM, Goldenberg R, Silver R, Smith GC, Pauli RM, Wapner RJ, Gardosi J, Pinar H, Grafe M, Kupferminc M, et al. Stillbirth classification-developing an international consensus for research: executive summary of a National Institute of Child Health and Human Development workshop. Obstet Gynecol 2009 Oct;114(4):901-914.

6. Flenady V, Koopmans L, Middleton P, Froen JF, Smith GC, Gibbons K, Coory M, Gordon A, Ellwood D, McIntyre HD, et al. Major risk factors for stillbirth in high-income countries: a systematic review and meta-analysis. Lancet $2011 \mathrm{Apr}$ 16;377(9774):1331-1340.

7. Villamor E, Cnattingius S. Interpregnancy weight change and risk of adverse pregnancy outcomes: a population-based study. Lancet 2006 Sep 30;368(9542):1164-1170.

8. Stephansson O, Dickman PW, Johansson AL, Kieler H, Cnattingius S. Time of birth and risk of intrapartum and neonatal death. Epidemiology 2003 Mar;14(2):218-222.

9. Waldenström U, Cnattingius S, Norman M, Schytt E. Advanced maternal age and stillbirth risk in nulliparous and multoparous women. Obstet Gynecol 2015 Aug;126(2):355-362.

10. McClure EM, Saleem S, Pasha O, Goldenberg RL. Stillbirth in developing countries: a review of causes, risk factors and prevention strategies. J Matern Fetal Neonatal Med 2009 Mar;22(3):183-190.

11. McPherson E. Discovering the cause of stillbirth. Curr Opin Obstet Gynecol 2013 Apr;25(2):152-156.
12. Korteweg FJ, Gordijn SJ, Timmer A, Erwich JJ, Bergmann KA, Bonman K, Ravise JM, Heringa MP, Hohn JP. The Tulip classification of perinatal mortality: introduction and multidisciplinary inter-rater agreement. BJOG 2006 Apr;113(4):393-401.

13. Kloos, K.; Vogel, M. Pathology of the perinatal period. Stuttgart: Thieme; 1974.

14. Joseph KS, Kinniburgh B, Hutcheon JA, Mehrabadi A, Dahlgren L, Basso M, Davies C, Lee L. Rationalizing definitions and procedures for optimizing clinical care and public health in fetal death and stillbirth. Obstet Gynecol 2015 Apr;125(4):784-788.

15. Stillbirth Collaborative Research Network Writing Group. Causes of death among stillbirths. JAMA 2011 Dec 14;306(22): 2459-2468.

16. Helgadóttir LB, Turowski G, Skjeldestad FE, Jacobsen AF, Sandset PM, Roald B, Jacobsen EM. Classification of stillbirths and risk factors by cause of death-a case-control study. Acta Obstet Gynecol Scand 2013 Mar;92(3):325-333.

17. Michalski ST, Porter J, Pauli RM. Costs and consequences of comprehensive stillbirth assessment. Am J Obstet Gynecol 2002 May;186(5):1027-1034.

18. Hübner J, Gast AS, Müller AM, Bartmann P, Gembruch U. Stillbirths in Germany: Retrospective analysis of 168 cases between 2003 and 2011. Z Geburtsh Neonatol 2015 Apr;219(2):73-80.

19. Royal College of Obstetricians and Gynecologists. Late intrauterine fetal death and stillbirth. Green-top guideline No. 55, Oct 2010.

20. Flenady V, Frøen JF, Pinar H, Torabi R, Saastad E, Guyon G, Russell L, Charles A, Harrison C, Chauke L. An evaluation of classification system of stillbirth. BMC Pregnancy Childbirth 2009 Jun 19;9:24.

21. Pinar H, Goldenberg RL, Koch MA, Heim-Hall J, Hawkins HK, Shehata B, Abramowsky C, Parker CB, Dudley DJ, Silver $\mathrm{RM}$, et al. Placental findings in singleton stillbirth. Obstet Gynecol 2014 Feb;123(2 Pt 1):325-336.

22. Dudenhausen JW, Grunebaum A, Staudinger UM. Optimization of women's health before conception when pregnancy has been postponed. J Perinat Med 2013 Jan;41(1):23-25. 